\title{
The TWIST/Mi2/NuRD protein complex and its essential role in cancer metastasis
}

\author{
Junjiang Fu ${ }^{1}$, Li Qin ${ }^{1}$, Tao He ${ }^{1,2}$, Jun Qin ${ }^{1}$, Jun Hong ${ }^{1}$, Jiemin Wong ${ }^{3}$, Lan Liao ${ }^{1}$, Jianming Xu ${ }^{1,2,3}$ \\ ${ }^{I}$ Department of Molecular and Cellular Biology, Baylor College of Medicine, One Baylor Plaza, Houston, TX 77030, USA; ${ }^{2}$ Lu- \\ zhou Medical College, Luzhou, Sichuan 646000, China; ${ }^{3}$ Institute of Biomedical Sciences and School of Life Sciences, East China \\ Normal University, Shanghai 200241, China
}

The epithelial-mesenchymal transition (EMT) converts epithelial tumor cells into invasive and metastatic cancer cells, leading to mortality in cancer patients. Although TWIST is a master regulator of EMT and metastasis for breast and other cancers, the mechanisms responsible for TWIST-mediated gene transcription remain unknown. In this study, purification and characterization of the TWIST protein complex revealed that TWIST interacts with several components of the Mi2/nucleosome remodeling and deacetylase (Mi2/NuRD) complex, MTA2, RbAp46, Mi2 and HDAC2, and recruits them to the proximal regions of the E-cadherin promoter for transcriptional repression. Depletion of these TWIST complex components from cancer cell lines that depend on TWIST for metastasis efficiently suppresses cell migration and invasion in culture and lung metastasis in mice. These findings not only provide novel mechanistic and functional links between TWIST and the Mi2/NuRD complex but also establish new essential roles for the components of $\mathrm{Mi} / \mathrm{NuRD}$ complex in cancer metastasis.

Keywords: TWIST; NuRD complex; gene repression; E-cadherin; epithelial-mesenchymal transition; metastasis Cell Research (2011) 21: 275-289. doi:10.1038/cr.2010.118; published online 17 August 2010

\section{Introduction}

The high incidence of various cancers is a major threat to human health. It is clear that the mortality of most cancer patients is not caused by primary tumors, but rather by its metastasis to other organs, such as bone, brain and internal organs. In the early stages of metastasis, certain epithelial tumor cells undergo epithelial-mesenchymal transition (EMT), which is characterized by the loss of epithelial polarity and differentiation markers such as E-cadherin and $\beta$-catenin and the gain of mesenchymal markers usually expressed by cells with a mesodermal origin, such as $\mathrm{N}$-cadherin, fibronectin and vimentin. Interestingly, loss of E-cadherin is necessary and sufficient to cause EMT $[1,2]$. The cancer cells with an EMT phenotype are capable of moving away from their parent tumor, interacting with stromal cells, invading their adja-

Correspondence: Jianming $\mathrm{Xu}$

Tel: +1-713-798-6199; Fax: +1-713-798-3017

E-mail: jxu@bcm.edu

Received 30 June 2010; revised 17 July 2010; accepted 20 July 2010; published online 17 August 2010 cent tissues and intravasating into the circulation systems [1]. Subsequently, the surviving cancer cells extravasate the circulation system, adapt themselves to the destination tissue environment and eventually develop into scattered metastatic tumors in distal organs. Since certain tumor cells, such as some breast tumor cells, often disseminate even before their parent tumor can be clinically diagnosed, it is important to explore the currently unclear molecular networks governing the above multi-step invasion-metastasis cascade.

TWIST is a master regulater of EMT and metastasis of breast cancer and its crucial role appears to be independent of another master regulator, Snail [3, 4]. TWIST is also a class II member of the basic helix-loop-helix (bHLH) transcription factor family, which is tissue-specifically expressed and binds E-box DNA elements as a homodimer or a heterodimer with a class I member, such as E12 or E47 [5]. During early embryonic development, TWIST is required for mesoderm induction [6]. In the process of metastasis, TWIST represses E-cadherin and $\beta$-catenin expression, promotes EMT, cell motility and invasiveness, and permits the intravasation of tumor cells [3, 7-9]. TWIST also enhances AKT2 expression, inhib- 
its p53 function and cell apoptosis, mediates HIF- $1 \alpha-$ enhanced cancer progression, promotes cell survival, and contributes to oncogenesis, angiogenesis and acquired Taxol resistance [7, 10-14]. In agreement with these critical roles in cancer cells, TWIST is overexpressed in breast, gastric, hepatocellular, prostate and bladder cancers, and its upregulation correlates with low E-cadherin expression, high cancer aggressiveness and poor survival rate $[3,8,15-19]$. These studies clearly demonstrate that TWIST is a master transcription factor that controls EMT, cancer progression and metastasis and a useful molecular target for treatment of cancer metastasis.

The Mi2/nucleosome remodeling and deacetylase (Mi2/NuRD) complex consists of multiple components and couples both histone deacetylase (HDAC) and chromatin-remodeling ATPase activities [20]. Its Mi2 $\alpha$ or Mi2 $\beta$ component contains ATPase activity that is stimulated by chromatin but not by free DNA or histones [20]. $\mathrm{Mi} 2$ is known to facilitate nucleosome mobility through a sliding mechanism [20-22]. The combined activities of HDAC and ATPase in the Mi2/NuRD complex result in the generation of densely packed, hypoacetylated nucleosomes for gene silencing [20]. The RbAp46 and/or RbAp48 subunits were originally identified as proteins associated with the retinoblastoma $(\mathrm{Rb})$ tumor suppressor [23], and they may function as structural proteins that provide interactive interfaces for other components of the Mi2/NuRD complex [20, 24, 25]. The Mi2/NuRD complex also contains one of the metastasis-associated (MTA) protein family members, MTA1, MTA2 or MTA3 [20, 26-28]. Each MTA member modifies the functional specificities of the Mi2/NuRD complexes relevant to its upstream and downstream signaling pathways and molecular targets. Specifically, MTA1 is expressed at high levels in metastatic cancer cells and its ectopic overexpression in mouse mammary epithelial cells can induce mammary epithelial proliferation and tumorigenesis [27, $29,30]$. In addition, MTA1 serves as a transcriptional corepressor of estrogen receptor $\alpha(\mathrm{ER} \alpha)$ [31]. MTA2 is also a corepressor of ER $\alpha$ and its overexpression leads to estrogen-independent growth of breast cancer cells [32]. MTA3 is an estrogen-inducible gene [20,33]. In contrast to MTA1, MTA3 represses the transcription of genes that are implicated in invasive growth, such as Snail [34]. Ectopic overexpression of MTA3 in the mouse mammary epithelial cells represses Wnt4 signaling and reduces mammary doctal branching [35]. Thus, different members of the MTA family direct the Mi2/NuRD complex to play distinct functions. The Mi2/NuRD complexes function primarily in gene repression involved in many biological processes, including cancer initiation and progression.
Although TWIST is recognized as a master regulator of cancer metastasis, the molecular mechanisms through which it regulates EMT and metastasis remain unclear. Furthermore, although certain components of the Mi2/ NuRD complex are known to play crucial roles in cancer, the molecular function of the complex has not been mechanistically linked to any master regulators that control EMT and metastasis. Similarly, the roles of MTA2 and $\mathrm{RbAp} 46$ in cancer require much more research. In this study, we purified and identified components of the TWIST protein complex. We show that TWIST is complexed with MTA2, RbAp46, Mi2 $\beta$ and HDAC2, which are key components of the Mi2/NuRD complex. We also provide compelling evidence that these components of the Mi2/MuRD complex are essential for TWISTmediated repression of E-cadherin expression, as well as cancer cell migration, invasion and metastasis.

\section{Results}

\section{Purification and identification of the TWIST protein com-} plex

To purify TWIST-associated proteins, inducible HEK293 cell lines expressing Flag-tagged TWIST (FTWIST) and control Flag (F) were generated (Figure 1A). After induction by doxycycline (DOX) for $6 \mathrm{~h}$, the proteins associated with F-TWIST and F were co-purified using immobilized Flag monoclonal antibody beads. The resulting protein complexes were separated in a gradient gel. In the stained gel, both multiple specific bands from the F-TWIST cells and several nonspecific bands from the control $\mathrm{F}$ cells were detected (Figure 1B). The gel slices with specific bands were excised, digested with trypsin and analyzed by mass spectrometry. In addition to TWIST, a number of other proteins, including TWIST2, E12/E47, HDAC2, RbAp46 and MTA2 (GenBank: NP_476527, NP_003191, NP_001518, NP_002884, and NP_004730), were identified (Figure 1B and data not shown). Among these proteins, TWIST2 and E12/ E47 are known heterodimeric proteins of TWIST [36, 37], while HDAC2, RbAp46 and MTA2, the essential components of the NuRD complex, are newly identified as TWIST-associated proteins. In mass spectrometry, the relative abundance of a protein can be represented by the index (\%) of relative peptide numbers, which is calculated by using the formula: (peptide hits/molecular weight $) \times 100$. In our analysis, the indexes of the relative peptide number for TWIST, E12/E47, MTA2, RbAp46 and HDAC2 were $262 \%, 7.3 \%, 9 \%, 6.3 \%$ and $3.6 \%$, respectively. Because TWIST was the protein directly pulled down by the antibody, it was expected to be much more abundant than other proteins in the purified protein 

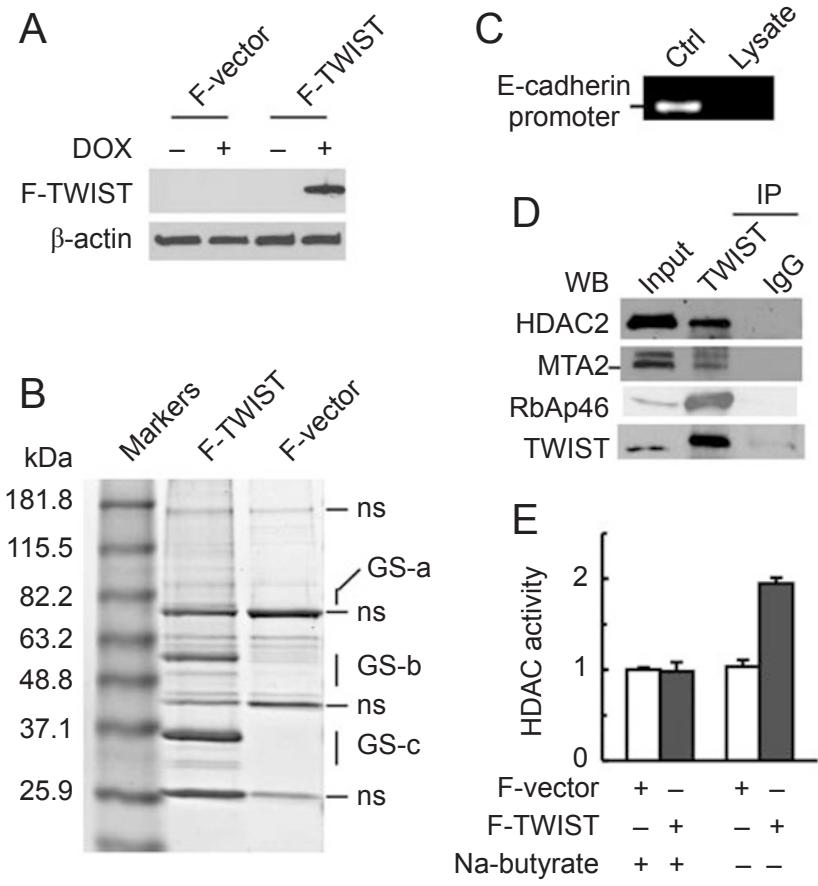

Figure 1 Purification and characterization of TWIST interacting proteins. (A) Induction of Flag-tagged TWIST (F-TWIST) protein. Stable HEK293 cells with F-TWIST or control F-vector were treated with vehicle or doxycyclin (DOX) for $6 \mathrm{~h}$. Western blot analysis was performed with whole-cell extracts and antibodies against Flag and $\beta$-actin. (B) Purification of TWIST protein complex. Flag antibody beads were incubated with F-TWIST and F-vector cell lysates. The immunopurified protein complexes were resolved by $4-20 \%$ SDS-PAGE and visualized by Imperial Protein Stain. Mass spectrometry analysis identified MTA2 and E12 proteins from the gel slice a (GS-a), HDAC2 and RbAp46 proteins from GS-b and TWIST1 and TWIST2 from GS-c of the F-TWIST gel lane. ns, non-specific bands appearing in both lanes. (C) The cell lysate was prepared in cell lysis buffer with $0.5 \%$ of NP-40 from 293 cells. The control (Ctrl) cell lysate was prepared with sonication for extracting genomic DNA as a positive control. PCR was performed to detect E-cadherin promoter in both cell lysates. (D) Co-IP and western blot. Endogenous TWIST protein in MDA-MB-435 cells was immunoprecipitated with TWIST antibody and control lgG. Associated proteins were assayed with indicated antibodies. (E) Detection of HDAC activity in TWIST protein complex. Chemical assay for HDAC activity was performed with $\left[\mathrm{H}^{3}\right]$-acetylated-histone $\mathrm{H} 4$ peptide as a substrate and co-immunoprecipitated materials by Flag antibody from F-TWIST and F-vector cells. The reactions were carried out with or without HDAC inhibitor sodium butyrate.

mixture. To examine whether DNA was involved in the purified TWIST protein complex, PCR was performed to detect an E-cadherin promoter region known to be associated with TWIST. The results showed that the DNA fragment spanning this region was undetectable in the cell lysate prepared in the cell lysis buffer with $0.5 \% \mathrm{NP}-$
40 for purifying TWSIT complex. In the positive control cell lysate prepared with sonication, the same DNA fragment was detected (Figure 1C). These results suggest that the purified TWIST protein complex was not linked together by DNA.

Furthermore, co-immunoprecipitation from cell extracts followed by immunoblotting verified that MTA2, RbAp46 and HDAC2 were also specifically associated with endogenous TWIST in MDA-MB-435 metastatic cancer cells (Figure 1D). In agreement with the presence of HDAC2 in the TWIST complex, a significant amount of sodium butyrate HDAC inhibitor-sensitive histone deacetylase activity was detected from the F-TWIST-associated proteins (Figure 1E). These results demonstrated that TWIST associates with the essential components, MTA2, RbAp46 and HDAC2, of the NuRD complex.

\section{TWIST interacts with multiple components of the NuRD complex}

To determine specific components of the NuRD complex that directly interact with TWIST, we produced glutathione-S-transferase (GST) and GST-TWIST fusion proteins in bacteria, as well as ${ }^{35} \mathrm{~S}$-labeled MTA2, RbAp46, HDAC2 and Mi2 $\beta$ (another known component of the NuRD complex) proteins by in vitro transcriptiontranslation-coupled reactions, and performed pull-down assays. The purified GST-TWIST, but not GST alone, pulled down MTA2, RbAp46 and Mi2 $\beta$, while both GST-TWIST and GST could not pull down HDAC2 (Figure 2A). Next, we generated and purified GSTTWIST-N and GST-TWIST-C fusion proteins with Nterminal 1-112 and C-terminal 110-202 amino acid (aa) residues of TWIST in order to determine how TWIST interacts with MTA2, RbAp46 and Mi2 $\beta$ (Figure 2B). The results showed that TWIST-N associated with MTA2 and Mi2 $\beta$, while TWIST-C associated with MTA2 and RbAp46 (Figure 2B). This indicates that MTA2 interacts with both N- and C-termini of TWIST, while Mi2 $\beta$ and $\mathrm{RbAp} 46$ interact with the $\mathrm{N}$-terminus and the $\mathrm{C}$-terminus of TWIST, respectively. However, since TWIST does not directly interact with HDAC2, they may associate through other components within the complex.

By using ${ }^{35} \mathrm{~S}$-labeled TWIST and GST-fusion proteins containing different fragments of MTA2, we also mapped the region of MTA2 responsible for the interaction with TWIST (Figure 2C and 2D). Although the N-terminus of MTA2 (aa 1-334) did not pull down TWIST, the Cterminus of MTA2 (aa 308-668) interacted strongly with TWIST (Figure 2D). Further analyses using GST-fusion proteins with different $\mathrm{C}$-terminal regions of MTA2 mapped the zinc-finger domain (aa 308-436) of MTA2 as a strong TWIST interaction domain, and the regions of 

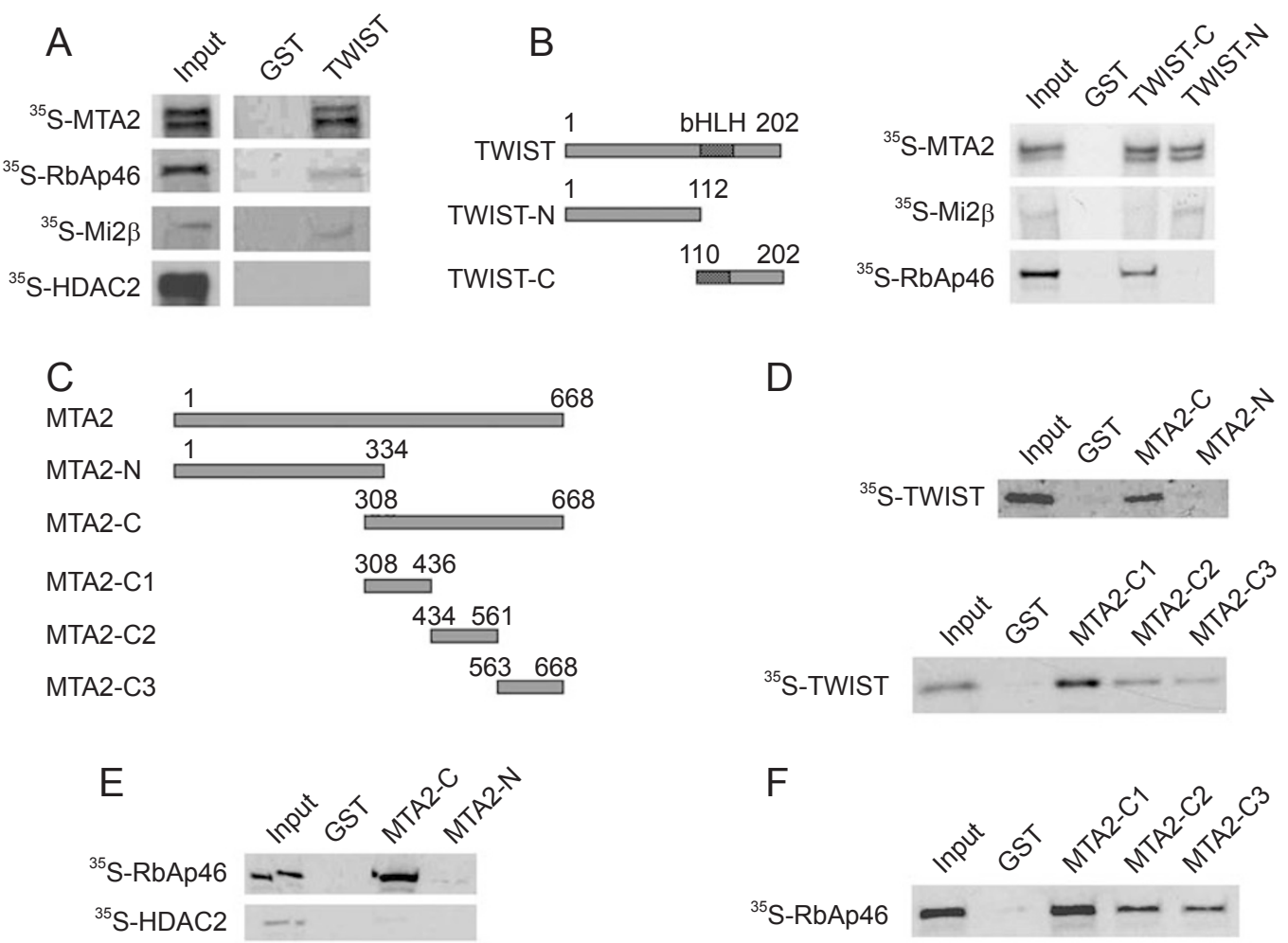

Figure 2 TWIST directly interacts with multiple components of the Mi2/NuRD/MTA2 complex. (A) GST pull-down assays. GST-TWIST and GST proteins purified from bacteria were mixed with ${ }^{35}$ S-labeled MTA2, Mi2, RbAp46 and HDAC2 proteins produced from transcription and translation-coupled reactions. The ${ }^{35} \mathrm{~S}$-labeled proteins bound to GST and GST-TWIST were resolved by SDS-PAGE and visualized by exposing to X-ray films. (B) Full-length TWIST and its N-and C-terminal fragments used in GST-fusion proteins and GST pull-down assays using GST, GST-TWIST-N and GST-TWIST-C and ${ }^{35}$ S-labeled MTA2, Mi2 and RbAP46 as indicated. (C) Full-length MTA2 and its fragments used in GST-fusion proteins. (D) GST pull-down assays using GST-fusion proteins containing MTA2 and its fragments and ${ }^{35}$ S-labeled TWIST. (E, F) GST pull-down assays using GST-fusion proteins containing MTA2 and its fragments and ${ }^{35}$ S-labeled RbAp46 and HDAC2.

aa 434-561 and aa 563-668 as weaker TWIST interaction domains (Figure 2D). Finally, in vitro interaction assays using the same set of GST-fusion proteins containing MTA2 and its fragments were performed to determine whether and where MTA2 interacted with HDAC2 and RbAp46. We found that the aa 308-668 region in MTA2 had a weak but detectable interaction with HDAC2 and a strong interaction with RbAp46 (Figure 2E). Further mapping revealed that this strong RbAp46 interaction domain was located in the aa 308-436 region in MTA2, although RbAp46 also interacted with the aa 434-668 region in MTA2 (Figure 2F). These results suggest that the TWIST-MTA2-RbAp46-Mi2-HDAC2 complex is maintained by multiple interactions between different components within the complex.

TWIST targets the NuRD complex to the E-cadherin promoter for repression

We performed several groups of experiments to as- sess the mechanisms by which the TWIST complex represses E-cadherin expression. First, by chromatin immunoprecipitation (ChIP) and ChIP-re-ChIP assays, we tested whether TWIST could recruit other components of the TWIST complex to the chromatin. The $5^{\prime}$ regulatory sequence of the human E-cadherin gene contains three E-boxes (Figure 3A). ChIP assays using HEK293 cells expressing F-TWIST or F revealed that TWIST was specifically associated with the DNA region containing the E-boxes but not with a distant control region of the 3' E-cadherin gene (Figure 3B). To test the association of other components of the complex with TWIST and the E-cadherin promoter, the immunoprecipitated materials associated with F-TWIST or F were subjected to the second-step ChIP using antibodies against Mi2 $\beta$, HDAC2 and MTA2. Results, with IgG as negative control, showed that Mi2 $\beta$, HDAC2 and MTA2 were indeed associated with TWIST and/or the E-cadherin promoter (Figure 3C). Although it cannot be absolutely excluded 
A

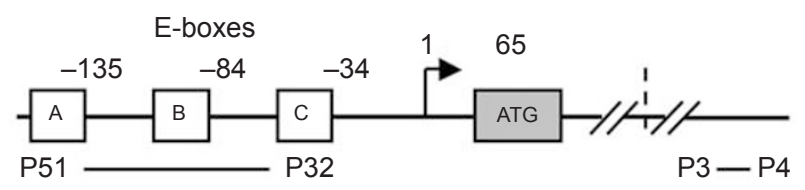

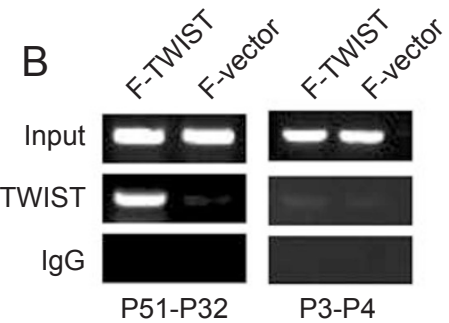

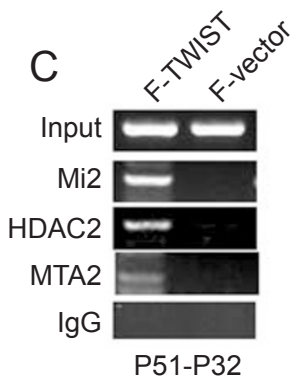

F

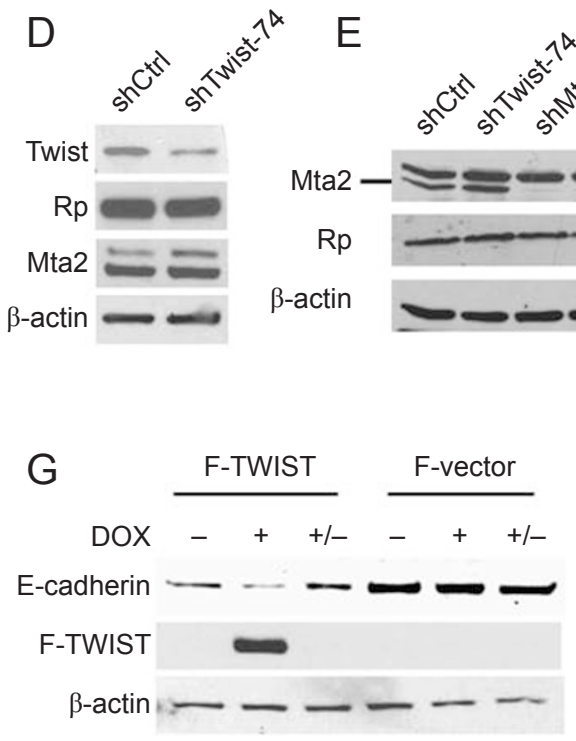

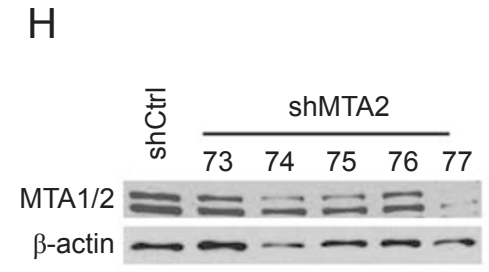

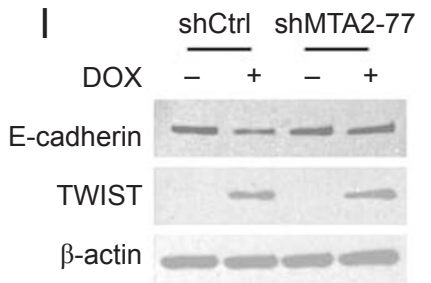

Figure 3 TWIST recruits the Mi2/NuRD/MTA2 complex to E-cadherin promoter and depends on MTA2 to repress E-cadherin expression. (A) The proximal region of the E-cadherin promoter contains three TWIST-binding E-boxes. In the ChIP assay, PCR with P51 and P32 primers was used to amplify the region with these E-boxes. PCR with primers P3 and P4 in the 3' region of the E-cadherin gene, which was more than $100 \mathrm{~kb}$ downstream from the indicated E-boxes, served as negative control in the ChIP assay. (B) ChIP assays using F-TWIST and F-vector HEK293 cells, Flag antibody or control lgG and primer pairs of P51/P32 and P3/P4 for E-cadherin gene. (C) ChIP-re-ChIP assays for the E-box region of the E-cadherin gene. The first-step immunoprecipitation was performed with Flag antibody and cross-linked and sonicated chromatin of F-TWIST and F-vector HEK293 cells. The second-step immunoprecipitation was performed with the eluted materials from the first step and with antibodies against Mi2, HDAC2 and MTA2 as indicated. IgG served as a negative control in the second step ChIP. (D, E) Generation of stable 4T1 cell pools with lentivirus-mediated expression of Twist shRNA (shTwist-74), Mta2 shRNA (shMta-54 and -56), RbAp46 shRNA (shRp-20 and -21) and non-targeting shRNA (shCtrl). Panel D shows efficient knockdown of Twist and the knockdown did not affect RbAp46 (Rp) and Mta2. Panel E shows efficient knockdown of Mta2 and RbAp46 proteins, and knockdown of any one of two proteins did not affect the other one (left panel) and also did not affect Twist (right panel). (F) Knockdown of Twist, Mta2 or RbAp46 in 4T1 cells increases E-cadherin promoter activity. 4T1 cell pools carrying nontargeting shRNA (control) and targeting shRNAs for Twist, Mta2 and RbAp46 as indicated were transfected with 100 ng of the E-Cad-promoter-Luciferase reporter plasmid and $30 \mathrm{ng}$ of $\beta$-gal expression plasmids in 24-well plates. Luciferase activity was assayed $40 \mathrm{~h}$ later, normalized to $\beta$-gal activity and presented as mean $\pm \mathrm{SD}, n=3$. (G) TWIST represses endogenous E-cadherin expression. HEK293 cells with F-TWIST and F-vector were set up in three groups. Groups 1 and 2 were treated with vehicle (lane 1 and 4) and DOX for 2 days to induce F-TWIST (lanes 2 and 3) and Flag (lanes 5 and 6). Group 3 was treated with DOX for 2 days, and then DOX was removed and cells were cultured for 7 more days (lanes 3 and 6). Western blot analyses were performed to analyze E-cadherin, F-TWIST and $\beta$-actin. (H) Western blot analysis of MTA2 in puromycinselected stable F-TWIST HEK293 cells infected by lentiviruses with a non-targeting control shRNA and five different shRNAs against human MTA2 mRNA. The shRNA-MTA2-77 lentivirus successfully reduced both MTA2 and MTA1 proteins. (I) MTA2 knockdown blocks TWIST-mediated suppression of E-cadherin expression. shRNA control and shRNA-MTA2-77 cells were treated with vehicle or DOX to induce F-TWIST for 3 days before western blot was performed to assay E-cadherin, F-TWIST and $\beta$-actin. 
that these proteins were co-precipitated through a DNA linkage, these results, together with the evidence showing direct interactions among TWIST, MTA2, RbAp46 and Mi2 $\beta$, suggest that these NuRD complex components are recruited to the E-cadherin promoter by TWIST.

Second, we examined how each individual component of the TWIST complex influences the E-cadherin promoter activity by shRNA lentivirus-based knockdown and transfection assays with an E-cadherin promoterdriven luciferase reporter. Twist, Mta2 and RbAp46 proteins were efficiently depleted by their respective shRNA lentiviruses but were not affected by the control shRNA lentivirus in the mouse 4T1 mammary tumor cells. Knockdown of Twist, Mta2 or RbAp46 had no significant effect on the protein levels of others (Figure 3D and $3 \mathrm{E})$. The strong lung metastatic feature of 4T1 cells has been shown to be dependent on Twist [3]. In agreement with previous studies showing that Twist suppressed Ecadherin promoter activity, its knockdown significantly released the inhibition on the E-cadherin promoter activity. More importantly, the knockdown of either Mta2 or RbAp46, each by two different shRNA lentiviruses, also released the inhibition of the E-cadherin promoter activity (Figure 3F).

Third, we investigated how TWIST complex regulates endogenous E-cadherin mRNA and protein expression. The control $\mathrm{F}$ cells had no F-TWIST expression regardless of DOX treatment, while untreated F-TWIST cells expressed a very low level of F-TWIST protein that could not be detected by standard western blot procedure (Figure $3 \mathrm{G}$ ) but could be detected by western blot with more protein loading and/or extended film exposure time (data not shown). The DOX-treated F-TWIST cells expressed F-TWIST protein as detected by western blot analysis (Figure 3G). Specifically, two-day induction significantly reduced E-cadherin mRNA and protein levels in the F-TWIST cells compared to the control F cells and un-induced F-TWIST. Interestingly, after DOX was removed from the medium for 7 days, cell F-TWIST proteins diminished while E-cadherin mRNA and protein levels recovered (Supplementary information, Figure S1 and Figure 3G). These results suggest that the TWISTdependent repression of E-cadherin expression is reversible. It should be noted that the E-cadherin level in FTWIST cells is lower than that in F cells even in the absence of DOX treatment. As these cells expressed a low level of F-TWIST even without DOX, the lower level of E-cadherin in F-TWIST cells could be a consequence of F-TWIST-mediated repression of E-cadherin expression. In correlation with the repression of E-cadherin expression by TWIST and promotion of EMT by TWIST, F-TWIST induction also repressed the expression of several other epithelial markers, including $\alpha$-cadherin, $\beta$-cadherin and $\gamma$-catenin. Similarly, when F-TWIST expression was blocked, the expression of these epithelial markers could be restored (Supplementary information, Figure S1).

Furthermore, to examine whether MTA2 is required for TWIST-mediated repression of E-cadherin expression, we generated F-TWIST cells with stable knockdown of MTA2. Among five shRNA constructs used to target different regions of human MTA2 mRNA, only shRNA-MTA2-77 efficiently knocked down MTA2 level, and also MTA1 level probably due to their sequence homology (Figure 3H). Importantly, although DOXinduced F-TWIST in shRNA-control F-TWIST cells significantly suppressed E-cadherin expression, DOXinduced F-TWIST in F-TWIST cells with MTA2 knockdown was unable to reduce E-cadherin levels (Figure 3I, compare lane 2 with lane 4). These results suggest that MTA2 is required for TWIST-mediated inhibition of Ecadherin expression. Overall, we conclude that TWIST interacts with multiple components of the Mi2/NuRD/ MTA2 complex and recruits this gene repression complex with HDAC activity to the E-cadherin promoter for repression of E-cadherin expression.

The components of TWIST complex are required for cancer cell migration and invasion

Cancer cell migration and invasion capabilities positively correlate with their metastatic capabilities and Twist plays an essential role in promotion of the mouse 4T1 mammary tumor cell migration, invasion and lung metastasis [3]. Thus, to evaluate the contributions of the Twist complex components in cancer cell migration and invasion, we infected 4T1 cells with either control nontargeting shRNA lentiviruses or specific shRNA lentiviruses that target Twist (shTwist-74), Mta2 (shMta2-54 and shMta2-56 against two regions of the MTA2 mRNA) and RbAp46 (shRbAp46-20 and shRbAp46-21 against two regions of the RbAp46 mRNA) mRNAs and established stable cell pools from each infection (Figure $3 \mathrm{D}$ and $3 \mathrm{E}$ ). Real-time measurements demonstrated that compared with shControl cells, the knockdown of Twist in shTwist-74 cells drastically reduced cell migration through a membrane with small pores and invasion through Matrigel, which was consistent with a previous study [3]. Interestingly, similar to the knockdown of Twist, the knockdown of Mta2 or RbAp46 equally reduced the migration and invasion capabilities of $4 \mathrm{~T} 1$ cells (Figure 4A and 4B). To exclude the possible influence of cell growth fluctuations on cell migration and invasion assays, we performed real-time cell growth measurements. Results showed that the shControl, 
shTwist-74, shRbAp46-20/-21 and shMta2-56 cell pools had similar growth rates, while the shMta2-54 cell pool showed a slightly lower growth rate (Figure 4C). Collectively, these results indicate that the components of the Twist complex are required for Twist-mediated 4T1 cell migration and invasion.

The MDA-MB-435 cell line expresses high-level TWIST [3] and is one of a few available human cancer cell lines that can efficiently metastasize to the lung in nude mice after formation of xenograft tumors in the mammary fat pads $[38,39]$. To test whether the TWIST complex also promotes cell migration, invasion and metastasis in this human cell line, shTWIST (one of the three tested constructs) and shRbAp46 (two of the three tested constructs) lentiviruses were used to generate stable MDA-MB-435 cell pools with largely reduced TWIST and RbAp46, respectively (Figure 4D and data not shown). Here, the cells infected with nontargeting shRNA (shControl) lentiviruses served as a control (Figure 4D). However, all available shMTA2 lentiviral constructs failed to knock down MTA2 in MDA-MB-435 cells (data not shown). In these stable cells, the tGFP indicator was concurrently expressed with shTWIST, shRbAp46 or shControl. The MDAMB-435 cell pools expressing shControl and shRbAp46 showed comparable growth rates, while knockdown of TWIST enhanced MDA-MB-435 cell growth (Figure 4E). Real-time measurements of cell invasion through a Matrigel layer revealed that knockdown of either TWIST or RbAp46 largely inhibited MDA-MB-435 cell invasion compared with shControl cells (Figure 4F). These results demonstrate that both TWIST and RbAp46, the
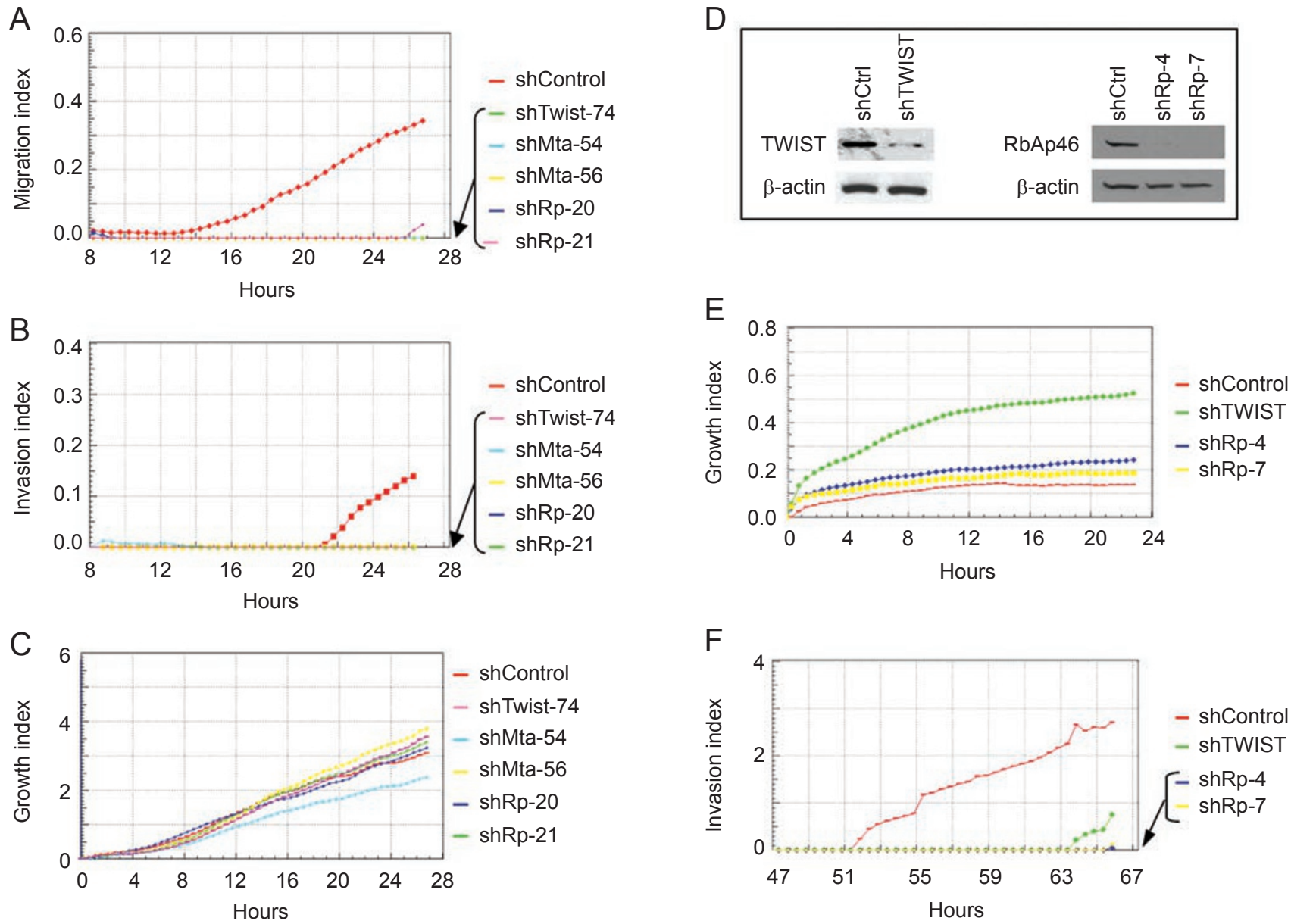

Figure 4 Knockdown of TWIST, MTA2 or RbAp46 specifically inhibits cancer cell migration and invasion. (A-C) Real-time assays of cell migration, invasion and growth for 4T1 cells with non-targeting shRNA (shControl) and targeting shRNAs specific to Twist (shTwist-74), Mta2 (shMta-54 and shMta-56) and RbAp46 (shRp-20 and shRp-21). (D) Western blot analysis for knockdown of TWIST and RbAp46 in MDA-MB-435 cells by shRNAs. (E, F) Real-time assays of cell growth and invasion for MDA-MB-435 cell pools expressing shControl, shTWIST and shRbAp46 (shRp-4 and shRp-7). 
two components of the TWIST complex, are required for MDA-MB-435 human cancer cells to invade the extracellular matrix.

The components of TWIST complex are required for cancer metastasis in vivo

Next, we assessed the roles of Twist complex components in breast tumor growth and metastasis using a congenic mouse tumor isograft model. Six stable 4T1 cell pools carrying shRNAs for control, Twist, Mta2 (two pools) and RbAp46 (two pools) (Figure 3D and 3E) were injected into the mammary fat pads of female BALB/c mice. Local tumor growth was measured by volume in the first 3 weeks and by tumor weight at the 4 th week experimental endpoint. All recipient mice developed local mammary tumors within a week and all tumors showed similar growth rates within the first 3 weeks. At the 4th week, the tumors formed from five of the six cell pools showed comparable weights, but tumors developed from the shRbAp46-21 cell pool were heavier than others (Figure 5A). Because the cell pool with RbAp46-20 shRNA did not change tumor growth, the increased growth rate here might be a non-specific effect of the RbAp4621 shRNA. In addition, all tumor tissues from different cell pools exhibited similar morphology of undifferentiated mammary carcinomas on H\&E-stained sections (data not shown). Collectively, these results suggest that Twist, Mta2 and RbAp46 are not required for local mammary tumor initiation and growth in mice.

Lung metastasis was analyzed by imaging the entire lung, counting the number of scattered macroscopic tumors on the surface and examining the H\&E-stained lung sections. Numerous metastatic large tumors were observed in the lungs of recipient mice with shControl mammary tumors. In contrast, both the sizes and numbers of metastatic lung tumors were drastically reduced in the recipient mice with shTwist, shMta and shRbAp46 mammary tumors (Figure 5B and 5C). Histological examination confirmed that compared with those in the mice carrying shControl mammary tumors, the number and sizes of metastatic lesions inside the lung were also remarkably reduced in the mice carrying shTwist, shMta2-54, shMta2-56, shRbAp46-20 and shRbAp46-21 mammary tumors (Figure 5D and data not shown). These results demonstrate that Mta2 and RbAp46, the two major components of the Twist complex, play essential roles in Twist-mediated breast cancer metastasis to the lung.

Cancer metastasis consists of multiple steps and Twist mainly promotes cancer cell invasion into the circulation system [3]. If the components of Twist complex work with Twist and mediate Twist-promoted metastasis, knockdown of these components should inhibit breast cancer cells from entering the circulation system. To test this hypothesis, we collected $150 \mu 1$ of blood samples from individual recipient mice carrying 4T1 tumors of similar sizes in their mammary fat pads and assayed the colony-forming units (CFUs) in a selective culture medium for tumor cell growth. Tumor cell colonies were formed from five out of seven blood samples of mice bearing shControl cell tumors and their average number of colonies was 24 per sample. In contrast, both the incidence of positive samples and the number of tumor cell colonies formed from blood samples of mice bearing shTwist, shMta2 and shRbAp46 tumors were significantly reduced. One out of 3,5 out of 10 , and 4 out of 10 blood samples from mice with shTwist, shMta2-54,

Figure 5 Twist, Mta2 and RbAp46 are required for breast cancer cell metastasis in mice. (A) Growth profiles of the isografted mammary tumors from the indicated $4 \mathrm{~T} 1 \mathrm{cells}$ in female BALB/c recipient mice. Data at the 2nd and 3rd weeks are presented as estimated average tumor volumes $\left(\mathrm{cm}^{3}\right)$. Data at the 4th week are presented as average tumor weight (g). (B) Representative photos of the lungs from different recipient mice carrying isografted mammary tumors from the indicated 4T1 cells at the 4th week. The asterisks indicate large metastatic tumors. (C) Average numbers of visible 4T1 metastatic nodules in individual mouse lung were counted and are presented as mean $\pm \mathrm{SD}, n=5$. (D) Representative H\&E-stained lung sections prepared from recipient mice bearing isografted mammary tumors formed from shControl, shMta2 and shRbAp46 4T1 cells. N, normal lung tissue; M, metastatic nodule. (E) Number of 4T1 tumor cell colonies formed in 15-day culture using $150 \mu$ l of blood collected from recipient mice bearing the indicated types of 4 T1 tumors for 4 weeks. (F) MDA-MB-435 xenograft tumor growth in athymic mice. MDA-MB-435 cells expressing shControl (shCtrl), shTWIST and shRbAp46 (shRp-4 and shRp-7) were injected into the mammary fat pads and tumor volumes were measured at the indicated time points. Ten tumors in each of the shControl, shTWIST and shRp-4 groups and eight tumors in the shRp-7 group were measured. (G) Representative tGFP images of primary tumors and lung metastasis derived from MDA-MB-435 cells with shControl, shTWIST and shRbAp46. H\&E-stained lung sections prepared from mice with the indicated types of tumors are also presented. $\mathrm{M}$, metastatic nodule; $\mathrm{N}$, normal lung tissue. $(\mathbf{H})$ Number of MDA-MB-435 lung tumors observed under a stereomicroscope. The average tumor number of the shControl group $(n=5)$ is significantly higher than that observed in the shTWIST $(n=5)$ and shRbAp46 $(n=9)$ groups $(P<0.05)$. (I) Number of tumor cell colonies formed in a culture containing $150 \mu \mathrm{l}$ of blood collected from recipient mice with the indicated types of MDA-MB-435 tumors. The average number of colonies formed from the blood of mice with shControl tumors is significantly higher than that formed from the blood samples of mice with shTWIST and shRbAp46 tumors $(P<0.05)$. 
A

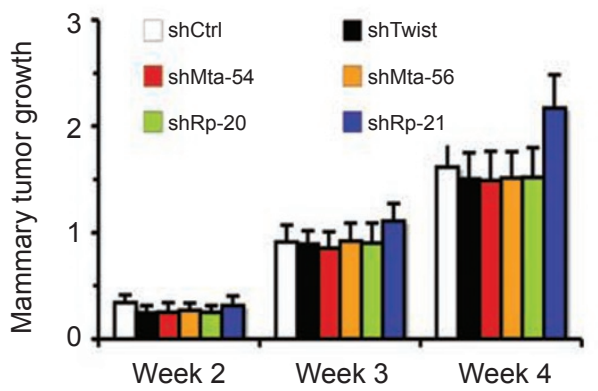

B

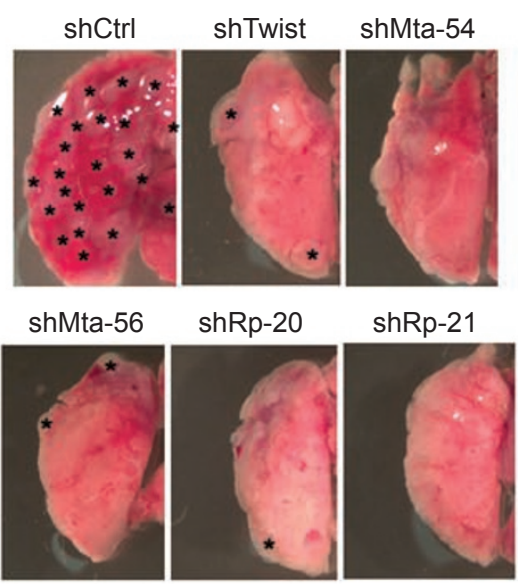

F

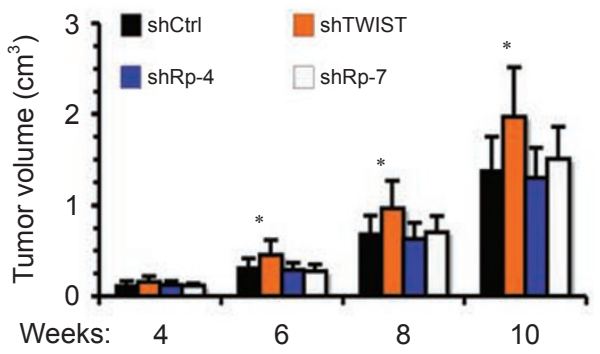

G

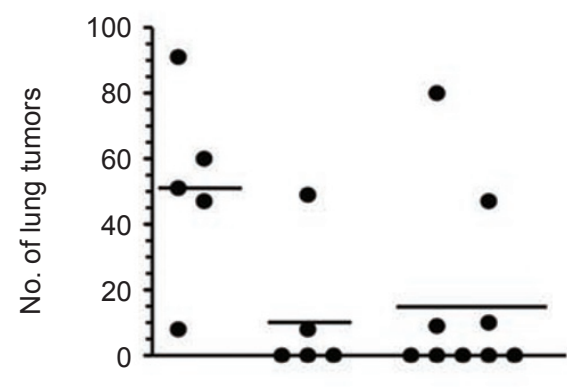

shRNA: Ctrl TWIST RbAp
C

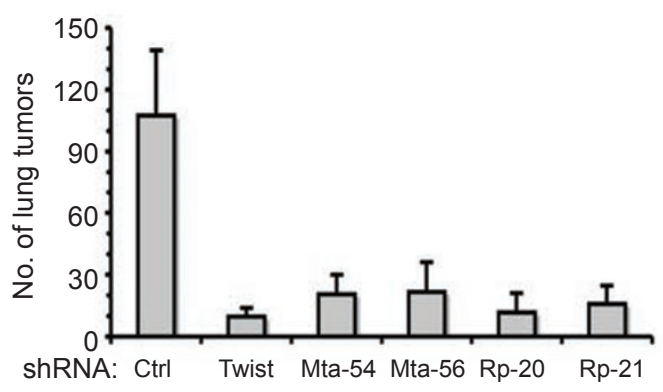

D

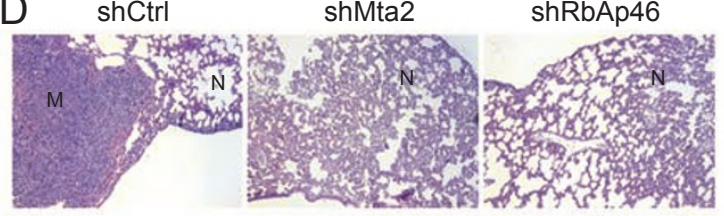

E

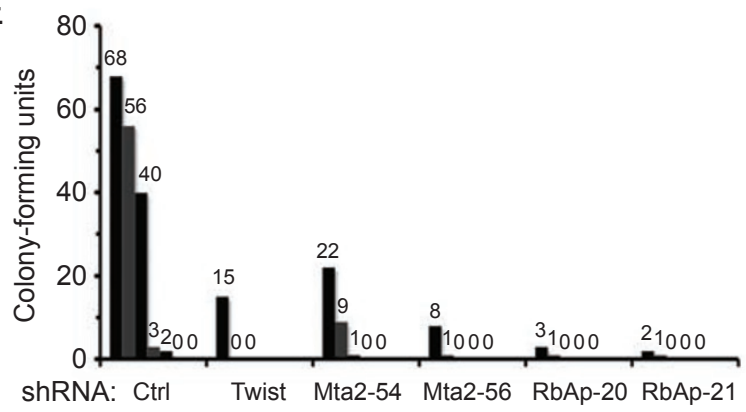

$\mathrm{H}$

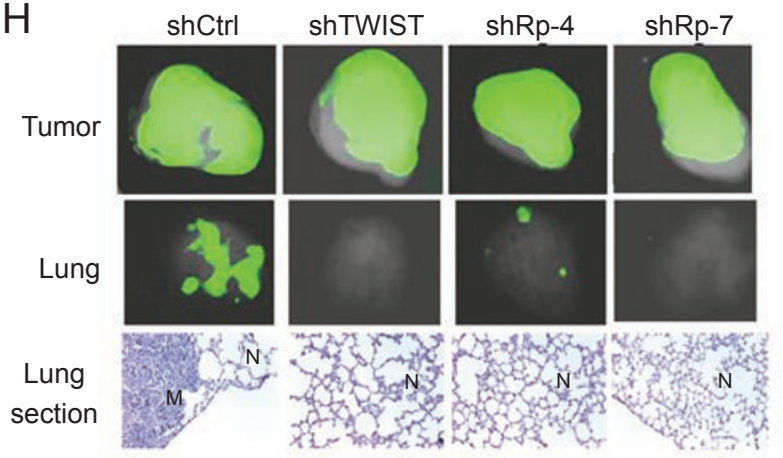

I

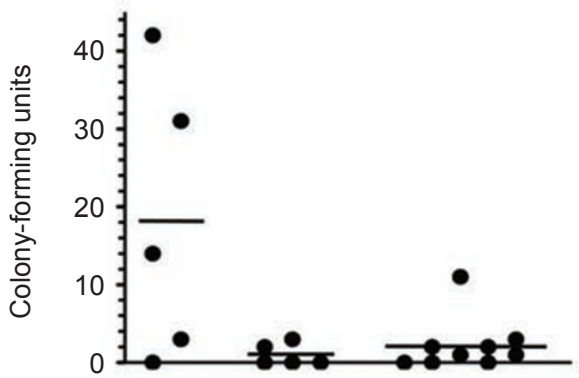

shRNA: Ctrl TWIST RbAp 
shMta2-56, shRbAp46-20 and shRbAp46-21 tumors developed tumor cell colonies in culture, and their average colony numbers per sample were 5, 6.4, 1.8, 0.8 and 0.6, respectively (Figure 5E). These results indicate that Mta2 and $\mathrm{RbAp} 46$ play crucial roles, similar to those by Twist, in the promotion of $4 \mathrm{~T} 1$ cancer cell intravasation.

To evaluate the role of the TWIST complex in metastasis derived from human cancer cells, we also injected tGFP-labeled shControl, shTWIST, shRbAp46-4 and shRbAp46-7 MDA-MB-435 cells (Figure 4D) into the mammary fat pads of nude mice. All mice developed local tumors within 2 weeks, and thereafter the tumor volume in each mouse was measured once a week. In agreement with their cell growth rates in culture (Figure $4 \mathrm{E}$ ), primary tumors derived from shControl cell pool and the two shRAp46 cell pools showed similar growth rates, while tumors derived from shTWIST cell pools were bigger, starting at 6 weeks after injection (Figure $5 \mathrm{~F}$ ). When tumor volume reached about $4 \mathrm{~cm}^{3}$ (the sum of both side tumors), the local tumor and lung were isolated and analyzed by tGFP imaging. All examined local tumors showed strong tGFP signal, validating the tumor origin from the injected cells. Interestingly, most lungs from mice with shControl tumors exhibited strong tGFP signals from the metastatic lung tumors, but most lungs from mice with shTWIST and shRbAp46 tumors did not show or showed weak tGFP signals (Figure $5 \mathrm{H}$ ). Consistent results were also obtained by examining lung sections and counting the number of metastatic lung tumors (Figure $5 \mathrm{H}$ and $5 \mathrm{G}$ ). The average numbers of lung tumors in mice with shTWIST or shRbAp46 tumors were significantly reduced in comparison with those in mice with shControl tumors (Figure 5G). Furthermore, both the frequency and the average number of cancer cell CFUs identified in the blood samples of mice with shControl tumors were much higher compared with that in the blood samples of mice with shTWIST or shRbAp46 tumors (Figure 5I). These results indicate that both TWIST and RbAp46 are required for the metastasis of MDA-MB-435 human cancer cells in vivo, especially for the cancer cells to invade into the circulation.

\section{Discussion}

TWIST interacts with multiple components of the Mi2/ NuRD gene repression complex

TWIST, a known master regulator of cancer metastasis, is a member of the bHLH transcription factor family $[4,5]$. TWIST forms homodimers or heterodimers with E12 or TWIST2 and binds the E-box DNA elements [5]. Although previous studies have suggested that TWIST can either activate or repress its target genes in breast cancer cells $[3,10]$, the mechanism through which TWIST regulates gene expression remains unknown. This lack of knowledge undermines our ability to understand specific transcriptional controls of cancer cell EMT and metastasis. To solve this problem, we focused on the purification and identification of the TWIST complex, which revealed that TWIST is specifically co-purified with TWIST2, E12, MTA2, RbAp46 and HDAC2. Among these TWIST-associated proteins, TWIST2 and E12 are known TWIST heterodimer partners [36, 37], indicating a suitable purification procedure. Interestingly, the other co-purified proteins contain components of the NuRD complex. The association between TWIST and these NuRD complex proteins was further confirmed by co-immunoprecipitation of endogenous proteins in MDA-MB-435 cells. As the complex contains HDAC2, it accordingly contains HDAC enzyme activities for histone deacetylation. Furthermore, the in vitro protein-protein interaction assays provided significant insights into the TWIST/Mi2/NuRD complex organizations. Specifically, the N-terminus of TWIST interacted with MTA2 and Mi2 $\beta$; the C-terminus of TWIST interacted with MTA2 and RbAp46; and there was no detectable interaction between TWIST and HDAC2. Further mapping revealed that the zinc-finger domain (aa 308-436) of MTA2 contained strong interaction motifs for both TWIST and RbAp46, while a downstream region of MTA2 might contain another weak interaction domain for TWIST. We also found a weak but detectable interaction between the C-terminus of MTA2 and HDAC2. These findings indicate that TWIST simultaneously interacts with multiple components (Mi2 $\beta$, RbAp46 and MTA2) and even with multiple domains of a single component (MTA2) of the Mi2/NurD complex, forming a relatively stable gene repression apparatus.

A potential concern for co-purified proteins that can associate with DNA is whether the co-purified protein components are associated through protein-protein interaction or protein-DNA-protein linkage. In our coimmunoprecipitation experiments, the cell lysis buffer with $0.5 \%$ of NP40 nonionic detergent could barely extract genomic DNA from the cells as assayed by PCR, so the purified TWIST-associated proteins should be a result of protein-protein interaction.

The interactive relationships between TWIST and components of the Mi2/NuRD complex are depicted in Figure 6. In this model, for the first time, we are able to observe the physical interactions between TWIST and Mi2 $\beta$, MTA2 and RbAp46, as well as the direct interactions between MTA2 and RbAp46 and between MTA2 and HDAC2. A recent study also showed the interaction between MTA1 and RbAp46 [40]. The inclusion of 


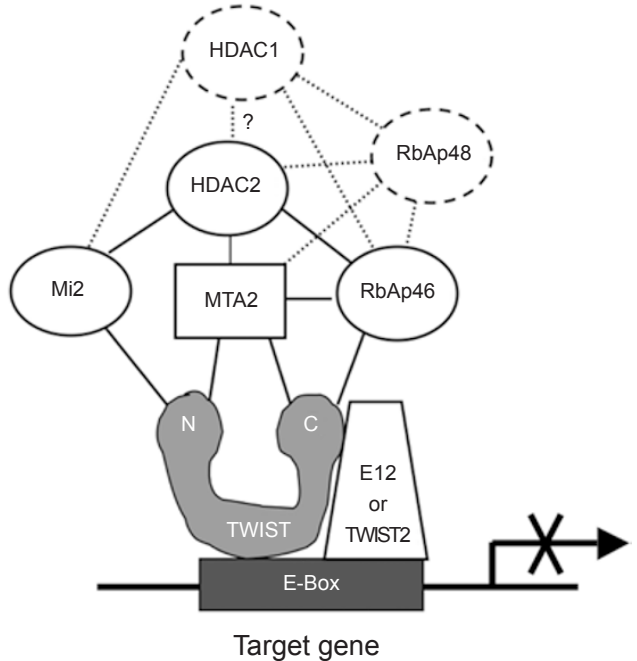

Figure 6 A model for TWIST-mediated gene repression. TWIST binds to E-box as homodimer or heterodimer with E12 or with TWIST2. The TWIST N-terminus interacts with MTA2 and Mi2, while its C-terminus interacts with MTA2 and RbAp46. Mi2, RbAp46 and MTA2 recruit HDAC2 to the DNA/TWIST complex through direct interactions. The TWIST-dependent recruitment of Mi2 and HDAC2 chromatin-remodeling enzymes to the promoter represses the target gene expression.

RbAp48 and HDAC1 in TWIST complex is based on previous studies that showed both as core components of the NuRD complex [41]. At this time, several unclear issues still deserve additional studies. First, it remains undetermined whether MTA1 could also associate with TWIST. Previous studies have shown that either MTA1 or MTA2, but not both, can exist in a single NuRD complex [28]. Second, although the co-purification of TWIST with TWIST2 and E12 suggests that TWIST/E12 and/or TWIST/TWIST2 heterodimers may be responsible for recruiting the Mi2/NuRD complex, the possibility that TWIST/TWIST homodimers might also play a role in recruitment of the Mi2/NuRD complex cannot be excluded. Third, previous studies showed that one of the methyl CpG-binding domain (MBD) family members, MBD2 or MBD3, also associates with the NuRD complex [20, 24, 42]. MBD2 can bind to methylated DNA for gene silencing, while MBD3 loses this function during vertebrate evolution [43]. Although neither MBD2 nor MBD3 was detected from the TWIST-copurified proteins by mass spectrometry, it is unclear whether MBD2 or MBD3 can associate with TWIST as a component of the Mi2/ NuRD complex. Future study on this issue may clarify the relationship between E-box/TWIST-mediated gene repression and DNA methylation/MBD2-mediated gene repression.
The TWIST/Mi2/NuRD/MTA2 complex represses gene expression by association with proximal promoter regions of its target genes

Although we know that TWIST represses E-cadherin promoter and the silencing of E-cadherin expression is a hallmark of EMT $[1,3,9]$, the specific mechanisms were unknown. In this study, we have provided multiple lines of evidence for the essential role of TWIST/Mi2/NuRD complex in the repression of the E-cadherin promoter. First, the ChIP-re-ChIP assays clearly indicate that the E-cadherin promoter associated not only with TWIST but also with Mi2 $\beta$, MTA2 and HDAC2. This suggests that TWIST may be capable of recruiting the Mi2/NuRD complex to the E-cadherin promoter region for histone modification, chromatin remodeling and transcriptional repression. Second, the knockdown of either MTA2 or RbAp46 increased the E-cadherin promoter activity as the knockdown of TWIST did, suggesting that the Mi2/ NuRD/MTA2 complex is required for TWIST-mediated repression of E-cadherin promoter activity. Of course, we could not exclude the possibility that knockdown of the NuRD complex components might also affect other transcription factors due to their diverse functions. Third, induction of TWIST in HEK293 cells decreased E-cadherin, while switch-off of TWIST restored E-cadherin. This implies the reversibility of TWISTdependent E-cadherin gene repression in these cells. More importantly, we showed that knockdown of MTA2 in these TWIST-induced cells efficiently blocked TWISTdependent E-cadherin gene repression. This indicates that the Mi2/NuRD compex components are required for TWIST-dependent E-cadherin gene repression. Thus, the above findings allow us to propose the regulatory pathway from TWIST to the formation of TWIST/Mi2/ NuRD complex, to the repression of E-cadherin promoter by Mi2 and HDAC-induced gene silencing, and to the cancer cell EMT and metastasis.

The TWIST/Mi2/NuRD/MTA2 complex regulates breast cancer metastasis

Using the 4T1 mouse tumor cell model, Yang et al. elegantly demonstrated the essential role of TWIST in metastasis [3]. The 4T1 cell line came from a metastatic subpopulation of the spontaneously arisen Balb/c mouse mammary tumor $[44,45]$. When $4 \mathrm{~T} 1$ cells were xenografted to the mammary glands of female Balb/c mice, the recipient mice rapidly formed local tumors and developed extensive lung metastasis. However, the knockdown of Twist in these 4T1 cells efficiently reduced their migration and invasive capabilities in culture and suppressed their lung metastasis in mice, indicating that 4T1 cells depend on Twist for metastasis [3]. Since this model has 
great advantages, including tumor cells with a natural origin and a highly metastatic feature, host mice with an intact immune system important for cancer metastasis, and a proven contribution of Twist to metastasis in these cells, it provides an ideal tool to examine the roles of other Twist complex components. Using this model, we compared the roles of Mta2 and RbAp46 with that of Twist. Our data indicated that the knockdown of Mta2 or RbAp46 had no specific effects on cell proliferation and local tumor growth but did inhibit 4T1 cell migration, invasion and metastasis to the same degree as knockdown of Twist did. In addition, similar to the inhibitory effect of Twist depletion on 4T1 tumor cell intravasation during metastasis, depletion of Mta2 or RbAp46 also drastically suppressed 4T1 tumor cell intravasation. These results strongly support that the Mi2/NuRD/MTA2 complex is essential to the mediation of Twist-controlled cancer cell migration, invasion and metastasis.

As TWIST promotes cancer cell intravasation from the local tumors [3], the cancer cells for testing TWIST function could not be injected into the blood. This considerably limited the choices of available metastatic models using human cancer cells. After examining multiple human cancer cell lines for their TWIST levels and tumorigenic and metastastic capacities, we decided to use the MBA-MD-435 cells that express high-level TWIST and exhibited nearly $100 \%$ tumorigenic and lung metastatic frequencies when injected into the mammary fat pads. MDA-MB-435 cells were previously used as a malignant human breast cancer cell line in numerous publications. However, its origin became ambiguous as its gene expression profile was found to be similar to M14 cells, which were believed to have a melanoma origin. Recently, Dr Chambers reviewed all the evidence and suggested that the MDA-MB-435 cell line is still a poorly differentiated, aggressive breast cancer line [46]. Regardless of its origin, our results demonstrate that the TWIST complex is required for the MDAMB-435 human cancer cells to invade ECM in vitro and metastasize in vivo, similar to its role in the 4T1 mouse breast cancer cells.

Off-targeting is a potential concern in all siRNA-based knockdown studies. Two quality control approaches are commonly used to validate the specificity of siRNAbased targeting. One is to express an siRNA-resistant mRNA in the knockdown cells as a rescue control, and the other is to use multiple siRNA constructs to target different mRNA resions to achieve consistent results. In this study, due to the hard-to-transfect feature of 4T1 and MDA-MB-435 cells, we chose to use multiple shRNA constructs in each knockdown experiment. For knocking down MTA2 and RbAp46, two shRNA constructs for human mRNAs and another two different shRNA constructs for mouse mRNAs were effective. For knocking down TWIST, although only one shRNA worked for human mRNA in HEK293 and MDAMB-435 cells and another shRNA worked for mouse mRNA in 4T1 cells, the effect of TWIST knockdown was predictable from previous studies, and shRNAs for human and mouse were different and targeted different regions of the mRNAs. The use of these multiple shRNA constructs in the knockdown experiments should efficiently avoid the potential off-targeting problem.

In conclusion, we have identified the TWIST protein complex that contains multiple components of the Mi2/ NuRD chromatin-remodeling and gene repression complex, including Mi2 $\beta$, MTA2, RbAp46 and HDAC2. These components of the Mi2/NuRD/MTA2 complex are responsible for mediating TWIST-repressed E-cadherin expression and TWIST-controlled cancer cell migration, invasion and metastasis. These findings allow us to propose a molecular regulatory pathway from TWIST to TWIST/Mi2/NuRD complex, to repression of E-cadherin promoter by HDAC and Mi2 enzyme activity-induced gene silencing, and to cancer cell EMT and metastasis. These findings also provide us more downstream components of the TWIST-signaling pathway as potential molecular targets to inhibit breast cancer metastasis.

\section{Materials and Methods}

\section{Inducible cell lines}

HEK293 cells with a Flp-In-T-REx system (Invitrogen) were transfected with the pcDNA5/FRT/TO empty vector and the vector containing the full-length hTWIST cDNA, respectively. Cells were cultured in medium containing $5 \mu \mathrm{g} / \mathrm{ml}$ blasticidin and $200 \mu \mathrm{g} / \mathrm{ml}$ hygromycin B for 10 days. The surviving cells were expanded and treated with $0.1 \mu \mathrm{g} / \mathrm{ml}$ of DOX to examine inducible expression of F-TWIST. The cells with empty vector were treated in the same way to serve as a control.

\section{Purification and identification of the TWIST complex}

F-TWIST and F-vector cells with $80 \%$ confluence were treated with DOX for $6 \mathrm{~h}$ and lysed in ice-cold EBC buffer containing 20 $\mathrm{mM}$ Tris- $\mathrm{HCl}$ ( $\mathrm{pH} 8.0$ ), $125 \mathrm{mM} \mathrm{NaCl}, 2$ mM EDTA 0.5\% NP40 and a protease inhibitor cocktail. The lysates were centrifuged at $16000 \times g$ for $1 \mathrm{~h}$ at $4{ }^{\circ} \mathrm{C}$. The supernatants were diluted 2 -fold with a buffer containing $20 \mathrm{mM}$ Tris- $\mathrm{HCl}$ (pH 8.0), $100 \mathrm{mM} \mathrm{KCl}$, $20 \%$ glycerol, $1 \mathrm{mM}$ EDTA and protease inhibitors and then incubated with the anti-Flag M2 agarose beads (Sigma) at $4{ }^{\circ} \mathrm{C}$ for $2 \mathrm{~h}$. The beads were washed 5 times in a buffer of $20 \mathrm{mM}$ Tris- $\mathrm{Cl}(\mathrm{pH}$ 8.0), $400 \mathrm{mM} \mathrm{KCl}, 0.5 \mathrm{mM}$ EDTA, 10\% glycerol, $0.25 \% \mathrm{NP}-40$ and protease inhibitors, and one time in TBS buffer with $50 \mathrm{mM}$ Tris- $\mathrm{HCl}(\mathrm{pH} 7.4)$ and $150 \mathrm{mM} \mathrm{NaCl}$. The bound proteins were eluted by $3 \times$ Flag peptide solution (Sigma), separated in a SDSPAGE gel and stained with Imperial Protein Stain (Pierce). Gel fragments with specific protein bands were incubated with trypsin 
and analyzed by mass spectrometry as described [47].

\section{HDAC activity assay}

HDAC activity assay kit was purchased from Upstate. The materials immunoprecipitated from equal amounts of $\mathrm{F}$ and F-TWIST cell lysates by Flag antibody beads were incubated with $10 \mu \mathrm{l}$ of ${ }^{3} \mathrm{H}$-acetate-labeled histone $\mathrm{H} 4$ peptide in $200 \mu \mathrm{l}$ of HDAC assay buffer containing $50 \mathrm{mM}$ Tris- $\mathrm{HCl}(\mathrm{pH} 8.0), 10 \%$ glycerol, $0.1 \mathrm{mM}$ EDTA and $1 \mathrm{mM}$ dithiothreitol for $2 \mathrm{~h}$ at $37{ }^{\circ} \mathrm{C}$. The reaction was stopped with $50 \mu \mathrm{l}$ of $1 \mathrm{~N} \mathrm{HCl}$ and $0.4 \mathrm{M}$ acetate. After centrifugation, $100 \mu \mathrm{l}$ of supernatant was collected, mixed with $3 \mathrm{ml}$ of scintillation cocktail and counted on a scintillation counter.

\section{Immunoprecipitation and immunoblotting}

Methods for immunoprecipitation (IP) and immunoblotting were described previously [47, 49]. TrueBlot IP/WB kit from eBioscience was used to eliminate the $\mathrm{IgG}$ bands in the immunoblotting analysis.

\section{GST pull-down assays}

GST, GST-TWIST and GST-MTA2 fusion proteins were expressed in BL21 Escherichia coli, solubilized in a sarkokyl solusion as described [50] and allowed to bind to the glutathionesepharose 4B breads (Amersham Pharmacia Biotech). ${ }^{35} \mathrm{~S}$-labeled TWIST, MTA2, HDAC2, Mi2 $\beta$ and RbAP46 proteins were synthesized using the TNT in vitro transcription-translation kit (Promega). The GST pull-down assays for protein-protein interactions between GST-fusion proteins and ${ }^{35} \mathrm{~S}$-labeled proteins were carried out as described [47].

\section{ChIP and ChIP-re-ChIP assays}

HEK293 F-TWIST and F-vector cells were cultured in 15$\mathrm{cm}$ plates to $65 \%$ confluence and treated with $0.1 \mu \mathrm{g} / \mathrm{ml} \mathrm{DOX}$ for $16 \mathrm{~h}$. The DNA-bound proteins in these cells were cross-linked in $1 \%$ formaldehyde for $10 \mathrm{~min}$. ChIP assays were performed using M2 Flag antibody beads and antibodies against TWIST, MTA2, HDAC2, RbBp46, Mi2b [49]. For ChIP-re-ChIP assays, the eluted materials were diluted with dilution buffer and subjected to the re-ChIP step using antibodies against MTA2, HDAC2 and Mi2 $\beta$ and the protein $\mathrm{A} / \mathrm{G}$ beads. PCR was performed to detect a 205bp proximal promoter region of the E-cadherin gene using primers ChIP51 (5'-cgt cta tgc gag gec ggg t-3') and ChIP3 (5'-aac tga ctt ccg caa gct cac a-3'). PCR amplification of a distant $3^{\prime}$ region of the E-cadherin gene with primers P54 (5'-gtc tga get ccc tga act cet c-3') and P34 (5'-cag caa cgt gat ttc tgc att t-3') was used as a negative control.

\section{Quantitative RT-PCR}

For quantitative RT-PCR (RT-qPCR), total RNA was extracted from cells and transcribed into cDNA as templates. Sequence-specific fluorescence-labeled probes and primers for TaqMan qPCR were matched by the Universal Probe Library Center software (Roche Applied Science). Relative concentrations of mRNA were obtained by normalization to 18 S RNA.

\section{Luciferase reporter assay}

$40 \%$-confluent cells in 24-well plates were transfected with 0.1 $\mu \mathrm{g}$ of the pGL3-Ecad(-108)-luc reporter and $30 \mathrm{ng}$ of $\beta$-Gal ex- pression plasmids using Lipofectamin 2000. Luciferase and $\beta$-gal activities were measured $40 \mathrm{~h}$ after transfection. The luciferase activity of each sample was normalized to the $\beta$-gal activity of the same sample. All experiments were performed in triplicates.

\section{shRNA-based knockdown of TWIST, MTA2 and RbAp46}

Four sets of shRNA lentiviral particles, each containing five shRNAs that target different regions of the same mRNA, were purchased from Sigma. These shRNAs target mouse Twist (SHVRSNM-11658), Mta2 (SHVRS-NM-11842) and RbAp46 (SHVRSNM-9031) mRNAs and human MTA2 (SHGLY-NM-004739) mRNA. In addition, one non-targeting shRNA lentivirus (SHC002V) was purchased as a control. 4T1 mouse breast tumor cells and HEK293 human cells were infected with these respective lentiviruses and selected in a medium containing $10 \mu \mathrm{g} / \mathrm{ml}$ of puromycin. Surviving cells were pooled and expanded for analyses of Twist, MTA2 and RbAp46 expression levels.

The GIPZ lentiviral shRNAmir-GFP system (Open Biosystems) was used to knock down human TWIST and RbAp46 in MDAMB-435 cells. Lentiviruses that co-express GFP with TWIST shRNAs (clone RHS4430-99329086), shRbAp46 shRNAs (clones RHS4430-98842683 and RHS4430-98893597) or non-targeting shRNA were used to infect MDA-MB-435 cells. The infected cells were selected in a medium containing $4 \mu \mathrm{g} / \mathrm{ml}$ of puromycin.

\section{Real-time assays of cell growth, migration and invasion}

Real-time cell growth, migration and invasion assays were carried out using an RT-CES system with 16-well E-plates and 16 double-layered chambers separated by a membrane with $8 \mu \mathrm{m}$ pores (ACEA Biosciences Inc.). For cell migration assay, the upper chambers were seeded with 50000 cells in $100 \mu$ of serumfree medium and the lower chambers were filled with medium containing 5\% serum. For cell invasion assay, the membrane was pre-coated with $20 \mu \mathrm{l}$ of 1:40 diluted Matrigel before cells were seeded. The process of cell migration and invasion was monitored every $30 \mathrm{~min}$ till the experimental endpoint.

\section{Analyses of tumor growth and metastasis in mice}

Mouse protocols were approved by the Baylor College of Medicine Animal Care and Use Committee. 8- to 12-week-old female BALB/cJ mice from Jackson Laboratory were anesthetized with $2.5 \%$ Avertin $(0.1 \mathrm{ml} / 10 \mathrm{~g}$, i.p.). For each recipient mouse, 4T1 mammary tumor cells $\left(2 \times 10^{5}\right)$ in $200 \mu$ of DME-10 medium were injected into the 4 th pair of mammary glands $(100 \mu \mathrm{l}$ for each gland) as described [51]. Tumor growth was measured weekly. Mice were sacrificed in 4 weeks. At the experimental endpoint, the weights of local mammary tumors were measured. The lung metastasis was carefully examined as described [51]. To determine the number of tumor cells in the blood, $150 \mu$ lof blood sample was collected from the heart of each mouse and cultured for 3 weeks in a selective medium for growth of puromycin-resistant 4T1 cells. The formed colonies were counted as described [3].

In another experiment, stable cell pools expressing tGFP and shRNAs for TWIST, RbAP46 or control shRNA were injected into the 4th mammary glands on both sides of 6-8-week-old female athymic nude mice from Harlan. For each site, $2 \times 10^{6}$ cells in $100 \mu \mathrm{l}$ PBS were injected. Tumor length $(L)$ and width $(W)$ were measured once a week and tumor volume was calculated as $(L \times$ $\left.W^{2}\right) / 2$. Mice were sacrificed when the total volume of both side 
tumors reached about $4 \mathrm{~cm}^{3} .150 \mu$ of blood sample was collected from the heart of each mouse for culture to determine the cancer cell CFUs in the circulation as described above. Primary tumors and lungs were isolated for imaging and histological analyses. The metastatic tumors appearing on the lung surface were counted under a stereomicroscope.

\section{Acknowledgments}

We thank D Liu, the director of the C-BASS Core of BCM, for providing shRNAmir-GFP-based lentiviruses. We thank $F$ Miller for 4T1 cells, E Fearon for the Ecad(-108)-luc plasmid, and Y Yuan, R Wu, K Shi, S Zhou and J Yang for discussion and experimental assistance and reagents. This work is partially supported by grants R01 CA112403, R01 CA119689 and R01 DK058242 to JX and U19 DK062434 to JQ from the National Institutes of Health, and RSG-05-082-01-TBE Scholar Award to JX from the American Cancer Society. We thank DL Duncan Cancer Center Pathway Discovery Core for protein complex identification.

\section{References}

1 Weinberg RA. The Biology of Cancer. New York: Garland Science, 2007.

2 Onder TT, Gupta PB, Mani SA, et al. Loss of E-cadherin promotes metastasis via multiple downstream transcriptional pathways. Cancer Res 2008; 68:3645-3654.

3 Yang J, Mani SA, Donaher JL, et al. Twist, a master regulator of morphogenesis, plays an essential role in tumor metastasis. Cell 2004; 117:927-939.

4 Yang J, Mani SA, Weinberg RA. Exploring a new twist on tumor metastasis. Cancer Res 2006; 66:4549-4552.

5 Castanon I, Baylies MK. A Twist in fate: evolutionary comparison of Twist structure and function. Gene 2002; 287:11-22.

6 O'Rourke MP, Tam PP. Twist functions in mouse development. Int J Dev Biol 2002; 46:401-413.

7 Mironchik Y, Winnard PT Jr, Vesuna F, et al. Twist overexpression induces in vivo angiogenesis and correlates with chromosomal instability in breast cancer. Cancer Res 2005; 65:10801-10809.

8 Kwok WK, Ling MT, Lee TW, et al. Up-regulation of TWIST in prostate cancer and its implication as a therapeutic target. Cancer Res 2005; 65:5153-5162.

9 Vesuna F, van Diest P, Chen JH, Raman V. Twist is a transcriptional repressor of E-cadherin gene expression in breast cancer. Biochem Biophys Res Commun 2008; 367:235241.

10 Cheng GZ, Chan J, Wang Q, et al. Twist transcriptionally upregulates AKT2 in breast cancer cells leading to increased migration, invasion, and resistance to paclitaxel. Cancer Res 2007; 67:1979-1987.

11 Maestro R, Dei Tos AP, Hamamori Y, et al. Twist is a potential oncogene that inhibits apoptosis. Genes Dev 1999; 13:2207-2217.

12 Valsesia-Wittmann S, Magdeleine M, Dupasquier S, et al. Oncogenic cooperation between H-Twist and N-Myc overrides failsafe programs in cancer cells. Cancer Cell 2004; 6:625-630.

13 Wang X, Ling MT, Guan XY, et al. Identification of a novel function of TWIST, a bHLH protein, in the development of acquired taxol resistance in human cancer cells. Oncogene 2004; 23:474-482.

14 Yang $\mathrm{MH}, \mathrm{Wu} \mathrm{MZ}$, Chiou $\mathrm{SH}$, et al. Direct regulation of TWIST by HIF-1alpha promotes metastasis. Nat Cell Biol 2008; 10:295-305.

15 Watanabe O, Imamura H, Shimizu T, et al. Expression of twist and wnt in human breast cancer. Anticancer Res 2004; 24:3851-3856.

16 Yan-Qi Z, Xue-Yan G, Shuang H, et al. Expression and significance of TWIST basic helix-loop-helix protein over-expression in gastric cancer. Pathology 2007; 39:470-475.

17 Lee TK, Poon RT, Yuen AP, et al. Twist overexpression correlates with hepatocellular carcinoma metastasis through induction of epithelial-mesenchymal transition. Clin Cancer Res 2006; 12:5369-5376.

18 Zhang Z, Xie D, Li X, et al. Significance of TWIST expression and its association with E-cadherin in bladder cancer. Hum Pathol 2007; 38:598-606.

19 Yuen HF, Chua CW, Chan YP, et al. Significance of TWIST and E-cadherin expression in the metastatic progression of prostatic cancer. Histopathology 2007; 50:648-658.

20 Denslow SA, Wade PA. The human Mi-2/NuRD complex and gene regulation. Oncogene 2007; 26:5433-5438.

21 Brehm A, Langst G, Kehle J, et al. dMi-2 and ISWI chromatin remodelling factors have distinct nucleosome binding and mobilization properties. EMBO J 2000; 19:4332-4341.

22 Guschin D, Wade PA, Kikyo N, Wolffe AP. ATP-dependent histone octamer mobilization and histone deacetylation mediated by the Mi-2 chromatin remodeling complex. Biochemistry 2000; 39:5238-5245.

23 Qian YW, Wang YC, Hollingsworth RE Jr, et al. A retinoblastoma-binding protein related to a negative regulator of Ras in yeast. Nature 1993; 364:648-652.

24 Zhang Y, Ng HH, Erdjument-Bromage H, et al. Analysis of the NuRD subunits reveals a histone deacetylase core complex and a connection with DNA methylation. Genes Dev 1999; 13:1924-1935.

25 Marhold J, Brehm A, Kramer K. The Drosophila methyl-DNA binding protein MBD2/3 interacts with the NuRD complex via p55 and MI-2. BMC Mol Biol 2004; 5:20.

26 Bowen NJ, Fujita N, Kajita M, Wade PA. Mi-2/NuRD: multiple complexes for many purposes. Biochim Biophys Acta 2004; 1677:52-57.

27 Manavathi B, Kumar R. Metastasis tumor antigens, an emerging family of multifaceted master coregulators. $J$ Biol Chem 2007; 282:1529-1533.

28 Yao YL, Yang WM. The metastasis-associated proteins 1 and 2 form distinct protein complexes with histone deacetylase activity. J Biol Chem 2003; 278:42560-42568.

29 Bagheri-Yarmand R, Talukder AH, Wang RA, Vadlamudi RK, Kumar R. Metastasis-associated protein 1 deregulation causes inappropriate mammary gland development and tumorigenesis. Development 2004; 131:3469-3479.

30 Toh Y, Nicolson GL. The role of the MTA family and their encoded proteins in human cancers: molecular functions and clinical implications. Clin Exp Metastasis 2009; 26:215-227.

31 Mazumdar A, Wang RA, Mishra SK, et al. Transcriptional repression of oestrogen receptor by metastasis-associated 
protein 1 corepressor. Nat Cell Biol 2001; 3:30-37.

32 Cui Y, Niu A, Pestell R, et al. Metastasis-associated protein 2 is a repressor of estrogen receptor alpha whose overexpression leads to estrogen-independent growth of human breast cancer cells. Mol Endocrinol 2006; 20:2020-2035.

33 Fujita N, Kajita M, Taysavang P, Wade PA. Hormonal regulation of metastasis-associated protein 3 transcription in breast cancer cells. Mol Endocrinol 2004; 18:2937-2949.

34 Fujita N, Jaye DL, Kajita M, et al. MTA3, a Mi-2/NuRD complex subunit, regulates an invasive growth pathway in breast cancer. Cell 2003; 113:207-219.

35 Zhang H, Singh RR, Talukder AH, Kumar R. Metastatic tumor antigen 3 is a direct corepressor of the Wnt4 pathway. Genes Dev 2006; 20:2943-2948.

36 Connerney J, Andreeva V, Leshem Y, et al. Twist1 dimer selection regulates cranial suture patterning and fusion. Dev Dyn 2006; 235:1345-1357.

37 Li L, Cserjesi P, Olson EN. Dermo-1: a novel twist-related bHLH protein expressed in the developing dermis. Dev Biol 1995; 172:280-292.

38 Price JE, Polyzos A, Zhang RD, Daniels LM. Tumorigenicity and metastasis of human breast carcinoma cell lines in nude mice. Cancer Res 1990; 50:717-721.

39 Price JE. Metastasis from human breast cancer cell lines. Breast Cancer Res Treat 1996; 39:93-102.

40 Roche AE, Bassett BJ, Samant SA, et al. The zinc finger and C-terminal domains of MTA proteins are required for FOG-2mediated transcriptional repression via the NuRD complex. $J$ Mol Cell Cardiol 2008; 44:352-360.

41 Feng Q, Zhang Y. The NuRD complex: linking histone modification to nucleosome remodeling. Curr Top Microbiol Immunol 2003; 274:269-290.
42 Le Guezennec X, Vermeulen M, Brinkman AB, et al. MBD2/ NuRD and MBD3/NuRD, two distinct complexes with different biochemical and functional properties. Mol Cell Biol 2006; 26:843-851.

43 Hendrich B, Tweedie S. The methyl-CpG binding domain and the evolving role of DNA methylation in animals. Trends $\mathrm{Ge}$ net 2003; 19:269-277.

44 Dexter DL, Kowalski HM, Blazar BA, et al. Heterogeneity of tumor cells from a single mouse mammary tumor. Cancer Res 1978; 38:3174-3181.

45 Aslakson CJ, Miller FR. Selective events in the metastatic process defined by analysis of the sequential dissemination of subpopulations of a mouse mammary tumor. Cancer Res 1992; 52:1399-1405.

46 Chambers AF. MDA-MB-435 and M14 cell lines: identical but not M14 melanoma? Cancer Res 2009; 69:5292-5293.

$47 \mathrm{Fu}$ J, Yoon HG, Qin J, Wong J. Regulation of P-TEFb elongation complex activity by CDK9 acetylation. Mol Cell Biol 2007; 27:4641-4651.

48 Lombaerts M, van Wezel T, Philippo K, et al. E-cadherin transcriptional downregulation by promoter methylation but not mutation is related to epithelial-to-mesenchymal transition in breast cancer cell lines. Br J Cancer 2006; 94:661-671.

49 Fu J, Jiang J, Li J, et al. Deleted in breast cancer 1, a novel androgen receptor (AR) coactivator that promotes AR DNAbinding activity. J Biol Chem 2009; 284:6832-6840.

50 Frangioni JV, Neel BG. Solubilization and purification of enzymatically active glutathione S-transferase (pGEX) fusion proteins. Anal Biochem 1993; 210:179-187.

51 Yuan Y, Qin L, Liu D, et al. Genetic screening reveals an essential role of p27kip1 in restriction of breast cancer progression. Cancer Res 2007; 67:8032-8042.

(Supplementary information is linked to the online version of the paper on the Cell Research website.) 